Algebraic $\&$ Geometric $\mathcal{T}_{\text {opology }}$

Volume 5 (2005) 379-403

Published: 22 May 2005

ATG

\title{
Experimental evidence for the Volume Conjecture for the simplest hyperbolic non-2-bridge knot
}

\author{
Stavros Garoufalidis \\ YUEHENG LAN
}

\begin{abstract}
Loosely speaking, the Volume Conjecture states that the limit of the $n$-th colored Jones polynomial of a hyperbolic knot, evaluated at the primitive complex $n$-th root of unity is a sequence of complex numbers that grows exponentially. Moreover, the exponential growth rate is proportional to the hyperbolic volume of the knot.

We provide an efficient formula for the colored Jones function of the simplest hyperbolic non-2-bridge knot, and using this formula, we provide numerical evidence for the Hyperbolic Volume Conjecture for the simplest hyperbolic non-2-bridge knot.
\end{abstract}

AMS Classification 57N10; 57M25

Keywords Knots, $q$-difference equations, asymptotics, Jones polynomial, Hyperbolic Volume Conjecture, character varieties, recursion relations, Kauffman bracket, skein module, fusion, SnapPea, m082

\section{Introduction}

\subsection{The Hyperbolic Volume Conjecture}

The Volume Conjecture connects two very different approaches to knot theory, namely Topological Quantum Field Theory and Riemannian (mostly Hyperbolic) Geometry. The Volume Conjecture states that for every hyperbolic knot $K$ in $S^{3}$

$$
\lim _{n \rightarrow \infty} \frac{\log \left|J_{K}(n)\left(e^{\frac{2 \pi i}{n}}\right)\right|}{n}=\frac{1}{2 \pi} \operatorname{vol}(K),
$$

where

- $J_{K}(n) \in \mathbb{Z}\left[q^{ \pm}\right]$is the Jones polynomial of a knot colored with the $n$ dimensional irreducible representation of $\mathfrak{s l}_{2}$, normalized so that it equals to 1 for the unknot (see [J], Tu] ), and 
- $\operatorname{vol}(K)$ is the volume of a complete hyperbolic metric in the knot complement $S^{3}-K$; see [Th].

The conjecture was formulated in this form by Murakami-Murakami [MM] following an earlier version due to Kashaev, $[\mathrm{K}$. The Volume Conjecture is an analytic question that contains two parts:

(a) to show that a limit of a sequence of complex numbers exists, and

(b) to identify the limit with a known geometric invariant of a knot.

Currently, the Volume Conjecture is known for only for the simplest hyperbolic knot: the $4_{1}$ (due to Ekholm), $[\underline{\mathrm{M}}]$. For the $4_{1}$ knot, due to special circumstances there is a 1-dimensional sum formula for $J_{4_{1}}(n)\left(e^{2 \pi i / n}\right)$ where the summand is a closed-form positive term. In that case, it is elementary to see that the volume conjecture holds. There is no other known knot that exhibits similar behavior.

Despite the optimism and the belief in the conjecture within the area of Quantum Topology, there is natural suspicion about it, due to lack of evidence.

There are several difficulties in the Volume Conjecture for a knot $K$ : the left hand side involves a sequence of polynomials $J_{K}(n)$ (for $n=1,2, \ldots$ ) with little understood relation to the geometry of the knot complement. In fact, a major problem is to give efficient multisum formulas for the polynomials $J_{K}(n)$. A naive approach based on cables computes $J_{K}(n)$ in $2^{c n^{2}}$ steps, where $n$ is the number of crossings. An alternative state-sum formula (see [GL, Section 3.2]) computes $J_{K}(n)$ in $n^{c}$ steps. In either case, when $c=18$ and $n=500$, these formulas are inefficient in numerically computing the left hand side of (1).

For a fixed knot, the polynomials $J_{K}(n)$ are not random. In GL, it was proven that they satisfy a linear $q$-difference equation. Moreover, it was explained that the results of GL together with the WZ algorithms (see [WZ]) can in principle compute the above mentioned linear $q$-difference equation. However computing the $q$-difference equation for knots with 6 crossings is already a difficult task. Thus, this method is of little use in numerical computations of the left hand side of (1).

In [Ga and [GG], the first author announced a program to prove the existence of the limit in the Volume Conjecture using asymptotics of solutions of linear $q$ difference equations. The main idea is that asymptotics of solutions of difference equations with a small parameter are governed by the average (on the unit circle) of the corresponding eigenvalues. When the eigenvalues do not collide or vanish, then this analysis was carried out in [GG]. In the Volume Conjecture, 
it is known that the eigenvalues collide (ven for the $4_{1}$ knot), and the analysis will be extended to cover this case in [CG]. Combined with the AJ Conjecture of $\mathrm{Ga}$, this program might identify the limit with the hyperbolic volume.

Among the hyperbolic knots, the 2-bridge knots are a tractable family with well-understood representation theory.

The purpose of the present paper is to provide:

- an efficient formula for the colored Jones function of the simplest hyperbolic non 2-bridge knot: the $k 4_{3}$ knot;

- using that formula, numerical evidence for the Volume Conjecture for the $k 4_{3}$ knot.

The key idea for efficient formulas for $J_{K}(n)$ is fusion as we explain below.

Aside from the above goals, we provide computation of numerous topological and geometric invariants of $k 4_{3}$, such as a presentation of its fundamental group, peripheral system, Alexander polynomial, $A$-polynomial, rank of Heegaard Floer Homology, volume of special Dehn fillings, invariant trace fields, as well as a multisum formula for the colored Jones function.

Let us end this introduction with an observation. It appears that for the knot $K_{0}=k 4_{3}$, the sequence that appears on the left hand side of Equation (1D) is eventually monotone decreasing. The same behavior is also exhibited for several 2-bridge knots that the authors tried. Monotonicity is an important clue for proving that the limit in the HVC exists. Let us formulate this as a conjecture:

Conjecture 1 For every knot $K$ in $S^{3}$, the sequence

$$
\frac{\log \left|J_{K}(n)\left(e^{\frac{2 \pi i}{n}}\right)\right|}{n}
$$

is eventually decreasing, and bounded above by zero.

\subsection{The knot $K_{0}$}

The word "simplest" does not refer to the number of crossings, but rather to the small number of ideal tetrahedra that are needed for an ideal triangulation of its complement.

Consider the twisted torus knot $K_{0}$, obtained from the torus knot $T(3,8)$ by adding a full positive twist to two strands. Actually, $K_{0}$ is the second simplest hyperbolic non-2-bridge knot with 4 ideal tetrahedra. The simplest hyperbolic 
non-2-bridge knot $k 3_{1}$ is the Pretzel knot $(-2,3,7)$ requiring 3 ideal tetrahedra, with same volume as the $5_{2}$ knot; see [CDW]. However, the colored Jones function of $k 3_{1}$ is rather complicated.

$K_{0}$ has braid presentation $b^{2}(a b)^{8}$, where $a=\sigma_{1}, b=\sigma_{2}$ are the standard generators of the braid group with 3 strands.

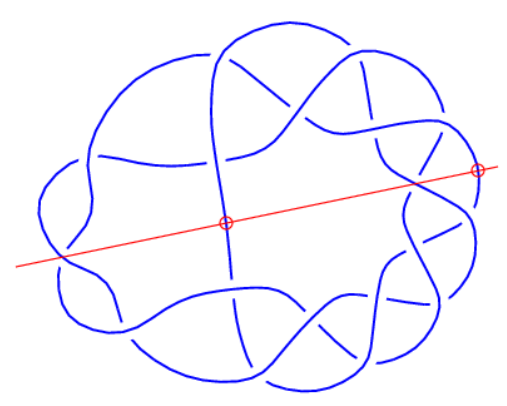

Figure 1: The knot $K_{0}$, and an involution which negates its meridian and longitude.

$K_{0}$ is a positive hyperbolic knot with 18 crossings. SnapPea identifies $K_{0}$ with the knot $k 4_{3}$ of the new census, also known as $m 082$ in the old census.

The notation $k 4_{3}$ reveals that $K_{0}$ is a hyperbolic knot whose complement can be triangulated with 4 hyperbolic ideal tetrahedra. It is the simplest hyperbolic non-2-bridge knot, as was discovered by [CDW].

$K_{0}$ belongs to a family of twisted torus knots, and this family populates the census of simplest hyperbolic manifolds other than the family of 2-bridge knots.

The Dowker code of $K_{0}$ is:

$\begin{array}{llllllllllllllllllll}18 & 1 & 14 & -16 & 18 & -20 & 22 & -24 & -26 & 28 & -30 & 32 & -34 & 36 & -12 & -2 & 4 & -6 & 8 & -10\end{array}$

$K_{0}$ has symmetry group $\mathbb{Z} / 2$. An involution that negates the meridian and longitude is shown in Figure 1.

\subsection{The quantum topology of $K_{0}$}

Let us define the quantum integer, the quantum factorial and the quantum binomial coefficients by:

$$
[n]=\frac{q^{n / 2}-q^{-n / 2}}{q^{1 / 2}-q^{-1 / 2}} \quad[n] !=[1][2] \ldots[n] \quad\left[\begin{array}{l}
n \\
k
\end{array}\right]=\frac{[n] !}{[k] ![n-k] !},
$$


where $[0] !=1$. The quantum binomial coefficients satisfy the following recursion relation:

$$
\left[\begin{array}{l}
n \\
k
\end{array}\right]=q^{-k / 2}\left[\begin{array}{c}
n-1 \\
k
\end{array}\right]+q^{(n-k) / 2}\left[\begin{array}{l}
n-1 \\
k-1
\end{array}\right]
$$

from which follows that $\left[\begin{array}{l}n \\ k\end{array}\right] \in \mathbb{Z}\left[q^{ \pm 1 / 2}\right]$.

For natural numbers $a, b$ with $b \leq a$, we denote

$$
[a, b] !=\frac{[a] !}{[b] !}
$$

which also lies in the ring $\mathbb{Z}\left[q^{ \pm 1 / 2}\right]$.

Definition 1.1 Let $J_{K}(n)$ denote the colored Jones polynomial of a knot $K$ in $S^{3}$ using the $n$-dimensional irreducible representation of $\mathfrak{s l}_{2}$, normalized to equal to 1 for the unknot; see [J, Tu].

The following theorem gives a triple sum formula for $J_{K_{0}}(n)$, where the summand is proper q-hypergeometric.

Theorem 1 For every natural number $n$, we have:

$$
\begin{aligned}
J_{K_{0}}(n+1) & \frac{1}{[n+1]} \sum_{k=0,2}^{2 n} \sum_{l=|n-k|, 2}^{n+k} \sum_{z}(-1)^{\frac{k}{2}+z} q^{-\frac{3}{8}\left(2 k+k^{2}\right)+\frac{7}{8}\left(2 l+l^{2}\right)-\frac{51}{8}\left(2 n+n^{2}\right)} \frac{[k+1][l+1]}{\left[\frac{2 n+k}{2}+1\right] !} \\
& {\left[\begin{array}{c}
\frac{k+l-n}{2} \\
\frac{n+2 k+l}{2}-z
\end{array}\right]\left[\begin{array}{c}
\frac{n+l-k}{2} \\
\frac{3 n+l}{2}-z
\end{array}\right]\left[\begin{array}{c}
\frac{n+k-l}{2} \\
\frac{2 n+2 k}{2}-z
\end{array}\right] } \\
& {\left[\frac{k}{2}\right] !^{2}\left[\frac{2 n-k}{2}, z-\frac{n+k+l}{2}\right] !\left[z+1, \frac{n+k+l}{2}+1\right] ! }
\end{aligned}
$$

Here $\sum_{k=a, 2}^{b}$ means summation for $k=a, a+2, \ldots, b$, for even $b-a$. Although the $z$ summation is infinite, only finitely many terms contribute. Explicitly, only the terms with $z \in \mathbb{N}$,

$$
\max \left(\frac{2 n+k}{2}, \frac{n+k+l}{2}\right) \leq z \leq \min \left(\frac{n+2 k+l}{2}, \frac{3 n+l}{2}, \frac{2 n+2 k}{2}\right)
$$

contribute.

Remark 1.2 The denominators in the above formula come from $[(2 n+k) / 2+$ $1]$ ! and $[n+1]$. When we evaluate the summand at $q=e^{2 \pi i /(n+1)}$, the order of the pole is 2 : 1 from $[(2 n+k) / 2+1]$ ! and 1 from $[n+1]$. 
Remark 1.3 In GL, Section 3.2] using properties of $R$-matrices, Le and the first author gave a canonical multisum formula for the colored Jones function of a knot which is presented as the closure of a braid. Specifically, if the braid has $c$ crossings, then the multisum formula involves summation over $c$ variables. In the case of the knot $K_{0}$, it involves summation over 18 variables, which makes it intractable, symbolically or numerically.

Remark 1.4 The well-known 3-term skein relation for the Jones polynomial

$$
q(\bigwedge)-q^{-1}(\searrow /)=\left(q^{1 / 2}-q^{-1 / 2}\right)() \circlearrowright
$$

allows one to compute naively the Jones polynomial of a knot with $c$ crossings in $2^{c}$ steps. By taking parallels, it allows one to compute the $n$-th colored Jones polynomial of a knot in $2^{c n^{2}}$ steps. For $n=500$, and $c=18$, this requires

$$
2^{18 \times 500^{2}} \approx 10^{1354634}
$$

terms.

\section{Numerical confirmation of the HVC for $K_{0}$}

Let

$$
\mathrm{VC}(n)=\left.\frac{2 \pi}{n} \log J_{K_{0}}(n)\right|_{q=e^{\frac{2 \pi i}{n}}}
$$

Using a fortran program by the second author (presented in the Appendix), we have computed $\operatorname{VC}(n)$ for $n$ up to 502 .

Here is a table of $\operatorname{Re}(\operatorname{VC}(n))$ versus $n$ :

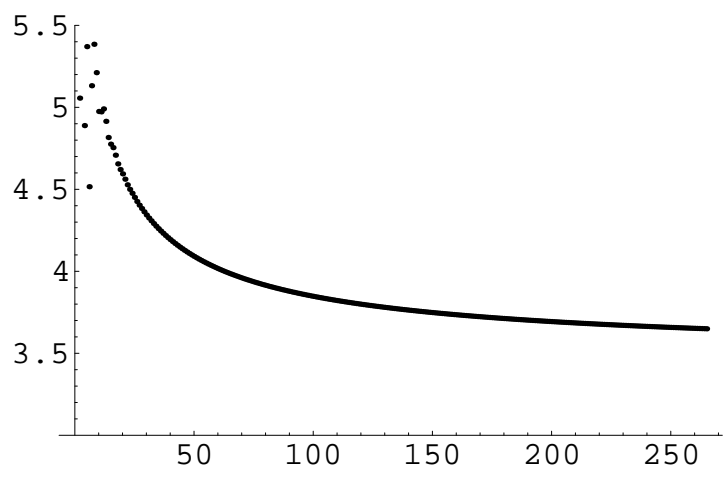


Here is a table of $\operatorname{Im}(\operatorname{VC}(n))$ versus $n$ :

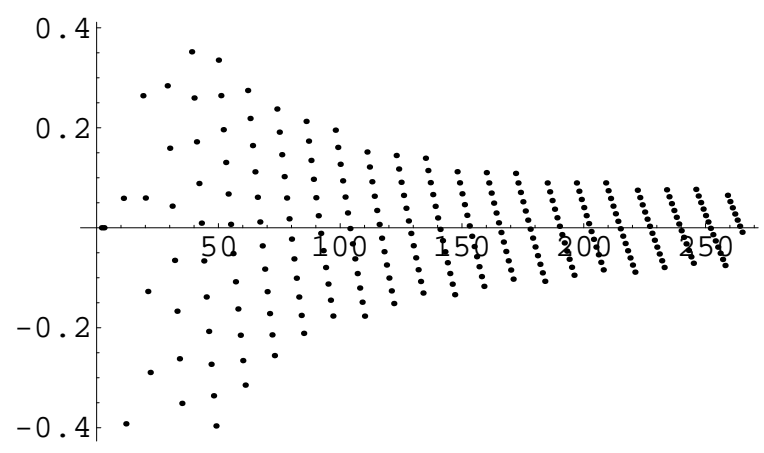

Here is a table of $\operatorname{Re}(\operatorname{VC}(n))$ versus $\operatorname{Im}(\operatorname{VC}(n))$ :

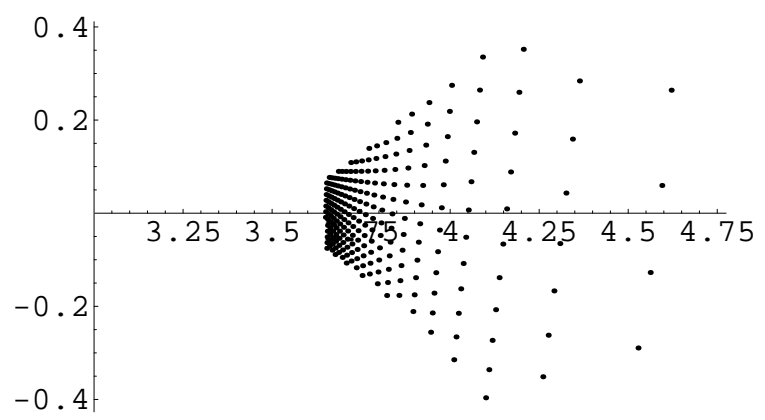

There is an evident 12 -fold periodicity that reflects the fact that $K_{0}$ is a twisted $(3,8)$ torus knot.

Quantum Field Theory predicts an asymptotic expansion of the form:

$$
J_{K_{0}}(n)\left(e^{2 \pi i / n}\right) \sim_{n \rightarrow \infty} e^{n \operatorname{vol}\left(K_{0}\right) /(2 \pi)+n i \operatorname{CS}\left(K_{0}\right)} n^{3 / 2}\left(C_{0}+\frac{C_{1}}{n}+\frac{C_{2}}{n^{2}}+\ldots\right)
$$

for constants $C_{i}$ (which depend on the knot $K_{0}$ ) with $C_{0} \neq 0$, and for $\operatorname{CS}\left(K_{0}\right) \in$ $\mathbb{R} / \mathbb{Z}$.

Fitting the data with least-squares for $n=21, \ldots, 250$ with $\log (n) / n, 1$ and $1 / n$ in Mathematica gives:

In $[1]:=$ Fit $[$ data $,\{\log [\mathrm{n}] / \mathrm{n}, 1,1 / \mathrm{n}\}, \mathrm{n}]$

gives

Out $[1]:=$

$3.4750687755045777-$

$5.518475184459029 / \mathrm{n}+9.282495203373793 \log [\mathrm{n}] / \mathrm{n}$

Algebraic 85 Geometric Topology, Volume 5 (2005) 
and plotting the data against the fit gives:

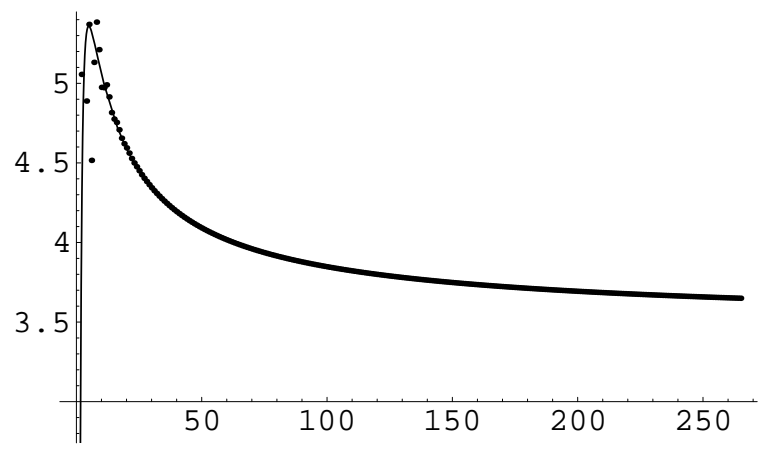

Here is a plot of the square of the error:

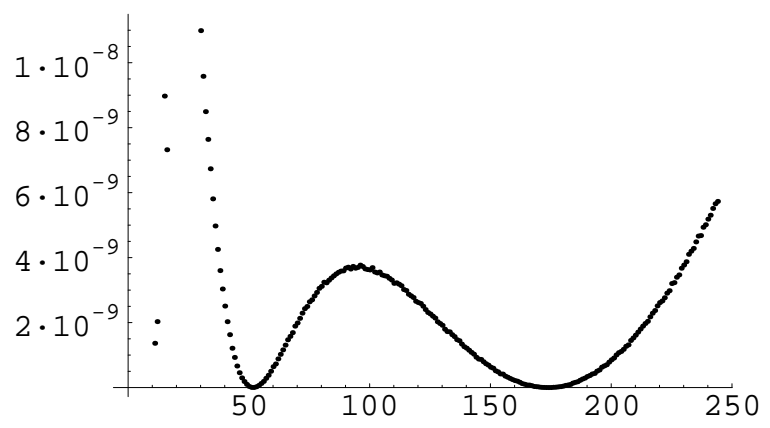

This shows that the error of the fit is within $10^{-4}$.

Notice that $\operatorname{vol}\left(K_{0}\right)=3.474247 \ldots$, and $9.282495203373793 /(2 \pi)=1.47735 \ldots$ Thus, the above data provides strong numerical evidence for the terms $\operatorname{vol}\left(K_{0}\right)$ and $3 / 2$ in Equation (3).

Here is a table of $\operatorname{Re}(\operatorname{VC}(2+10 n))$ versus $n$ for $n=0, \ldots, 50$ :

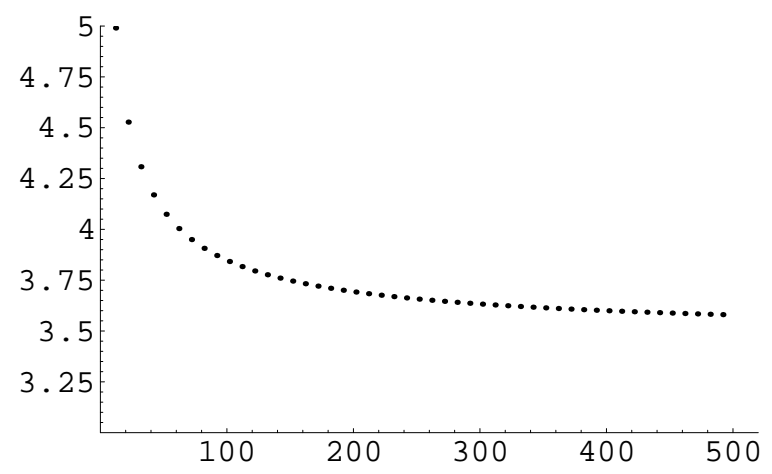

Algebraic 83 Geometric Topology, Volume 5 (2005) 
Here is a table of $\operatorname{Im}(\operatorname{VC}(2+10 n))$ versus $n$ for $n=0, \ldots, 50$ :

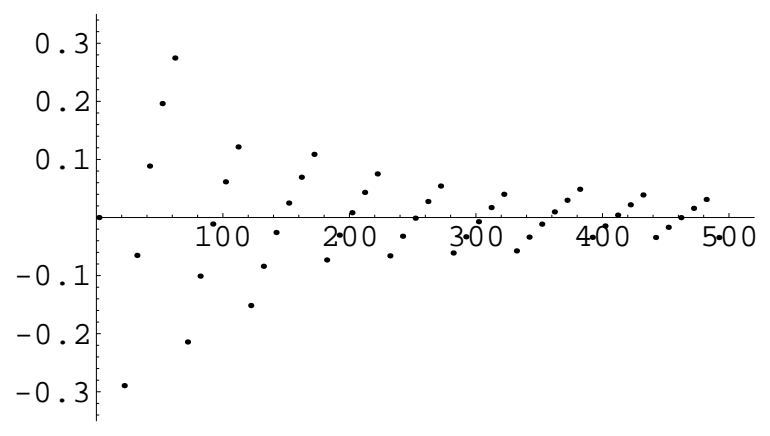

Here is a table of $\operatorname{Re}(\operatorname{VC}(n))$ versus $\operatorname{Im}(\operatorname{VC}(2+10 n))$ for $n=0, \ldots, 50$ :

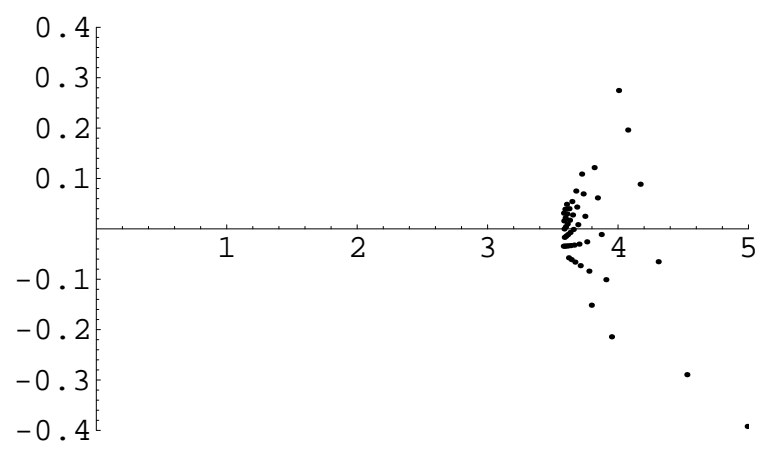

\section{Proofs}

\subsection{Fusion}

The colored Jones function $J_{K}(n) \in \mathbb{Z}\left[q^{ \pm}\right]$of a knot $K$ in $S^{3}$ was originally defined using $R$-matrices, that is solutions to the Yang-Baxter equations; see [J] and $[\mathrm{Tu}$. These solutions are intimately related to the representation theory of quantum groups. In GL Le and the first author used the theory of $R$-matrices to give formulas for the colored Jones function of a knot $K$. Given a braid presentation $\beta$ of a knot $K$ (that is, a word of length $c$ in the braid group of some number of strands), one constructs a proper $q$-hypergeometric function $F_{\beta}\left(n, k_{1}, \ldots, k_{c}\right)$ such that

$$
J_{K}(n)=\sum_{k_{1}, \ldots, k_{c}=0}^{\infty} F_{\beta}\left(n, k_{1}, \ldots, k_{c}\right) .
$$


The good thing is that for fixed $n \in \mathbb{N}$, only finitely many terms are nonzero. Moreover, the function $F_{\beta}$ takes values in the ring $\mathbb{Z}\left[q^{ \pm 1 / 2}\right]$, i.e., it has no denominators. Thus, we can commute summation and evaluation at an complex $n$-th root of unity.

The bad thing is that the above sum is multidimensional, which makes evaluation, symbolic or numerical, impractical.

Thus, we have to find alternative form of presenting $J_{K}(n)$. To achieve this, we will use the Kauffman bracket skein module, and fusion. The latter molds together pieces of a knot, producing knotted trivalent graphs. An advantage of fusion is that it deals nicely with twists (see the figures below), and thus it cuts down on the number of summation variables. A disadvantage of fusion is that it produces denominators which vanish when we evaluate at complex $n$th roots of unity. So, fusion gains low number of summation variables, at the cost of producing denominators. Of course, if one expands out all the terms, then the denominators will eventually cancel out, since the colored Jones function is a Laurent polynomial. However, expanding out denominators and canceling is too costly, and not efficient enough. The programs developed by the second author demonstrate that we can control fusion numerically, even when evaluating at complex roots of unity.

\subsection{Review of the Kauffman bracket of knotted trivalent graphs}

Let us recall what is fusion. A standard reference for this section is MV and $[\mathrm{KL}$. We will work with the Kauffman bracket skein module of links, summarized in the following figure:

$$
\left.\searrow /\left(A^{\smile}+A^{-1}\right)(, \quad) \bigcirc=-\left(A^{2}+A^{-2}\right)\right)
$$

Here and below, $A=q^{1 / 4}$. The proof of Theorem 1] will use fusion, leading to knotted trivalent graphs; the latter are framed embedded colored trivalent graphs. Let us recall the basic rules of fusion, from [MV] (see also $[\mathrm{KL}$ ):

$$
\begin{gathered}
{ }^{i}|\quad|{ }^{j}=\sum_{k} \frac{\langle k\rangle}{\langle i, j, k\rangle}{ }_{i}^{i} Y_{j}^{j} \\
Y_{c}^{b}=\left.\delta(c ; a, b)^{a} Y_{c}^{b} \quad a\right|_{\rho} ^{b}=\mu(a)^{a} \mid
\end{gathered}
$$


Here, $\langle i, j, k\rangle$ is defined below and

$$
\begin{aligned}
\langle k\rangle & =(-1)^{k}[k+1]=(-1)^{k} \frac{A^{2 k+2}-A^{-2 k-2}}{A^{2}-A^{-2}} \\
\mu(k) & =(-1)^{k} A^{k^{2}+2 k} \\
\delta(c ; a, b) & =(-1)^{k} A^{i j-k(i+j+k+2)} .
\end{aligned}
$$

With the use of fusion one can reduce the computation of knotted trivalent graphs to the values of a standard trihedron and tetrahedron.

Following standard skein theory conventions, a triple $(a, b, c)$ of nonnegative integers is called admissible if $a+b+c$ is even and $|b-c| \leq a \leq b+c$. Given an admissible triple, let $i=(b+c-a) / 2, j=(a+c-b) / 2$ and $k=(a+b-c) / 2$ denote the corresponding internal colors. Of importance are the values of a colored trihedron

$$
\frac{a}{c}=\langle a, b, c\rangle
$$

and tetrahedron

$$
\overbrace{A}^{B} C_{F}^{A}=\left\langle\begin{array}{lll}
A & B & E \\
D & C & F
\end{array}\right\rangle \text {. }
$$

Using the notation of Masbaum-Vogel [MV] and also Kauffman-Lins [KL], the trihedron and tetrahedron coefficients are given by:

$$
\begin{aligned}
& \langle a, b, c\rangle=(-1)^{i+j+k} \frac{[i+j+k+1] ![i+j] ![i+k] ![j+k] !}{[a] ![b] ![c] !} \\
& \left\langle\begin{array}{lll}
A & B & E \\
D & C & F
\end{array}\right\rangle=\frac{\prod_{i=1}^{3} \prod_{j=1}^{4}\left[b_{i}-a_{j}\right] !}{[A] ![B] ![C] ![D] ![E] ![F] !}\left(\begin{array}{lllllll}
a_{1} & & a_{2} & & a_{3} & & a_{4} \\
& b_{1} & & b_{2} & & b_{3} &
\end{array}\right),
\end{aligned}
$$

where

- we assume that the triples $(A, B, E),(B, D, F),(E, D, C)$ and $(A, C, F)$ are admissible,

- $\Sigma=A+B+C+D+E+F$,

$$
\begin{array}{ll}
a_{1}=(A+B+E) / 2 & b_{1}=(\Sigma-A-D) / 2 \\
a_{2}=(B+D+F) / 2 & b_{2}=(\Sigma-E-F) / 2 \\
a_{3}=(C+D+E) / 2 & b_{3}=(\Sigma-B-C) / 2 \\
a_{4}=(A+C+F) / 2, &
\end{array}
$$


- and

$$
\left(\begin{array}{ccccccc}
a_{1} & & a_{2} & & a_{3} & & a_{4} \\
& b_{1} & & b_{2} & & b_{3} &
\end{array}\right)=\sum_{\max \left(a_{j}\right) \leq \zeta \leq \min \left(b_{i}\right)} \frac{(-1)^{\zeta}[\zeta+1] !}{\prod_{i=1}^{3}\left[b_{i}-\zeta\right] ! \prod_{j=1}^{4}\left[\zeta-a_{j}\right] !}
$$

\subsection{Proof of theorem 1}

Recall that $K_{0}$ is the closure of the braid $b^{2}(a b)^{8}=b a^{-1}(a b)^{9}=b a^{-1} c^{3}$ where $c=(a b)^{3}$ represents a full twist. $c$ can be obtained by first giving a full twist on the first two strands, and then a full twist of the third strand around the first two. Thus, $K_{0}$ is obtained from the closure of the following figure:

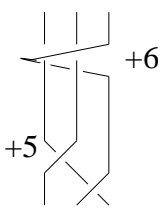

We want to compute the Kauffman bracket $\left\langle K_{0}, n\right\rangle$ colored by $n$, using the zero framing of $K$; this differs from the blackboard framing by +18 . Now, let us fuse the first two strands, and undo the +5 half twists. We obtain that

$$
\left.\left.\left\langle K_{0}, n\right\rangle=\mu(n)^{-18} \sum_{k} \frac{\langle k\rangle}{\langle n, n, k\rangle}+\frac{\mid}{k}\right\rangle\right]^{+6}=\sum_{k} \frac{\langle k\rangle}{\langle n, n, k\rangle} \delta(k ; n, n)^{5}{ }^{\frac{\mid}{k}}+6
$$

where the color of any noncolored edges is $n$. Let us isotope the fused strand above the +6 half twists, fuse again, and then undo the +6 half-twists. We obtain that

$$
\left\langle K_{0}, n\right\rangle=\mu(n)^{-18} \sum_{k, l} \frac{\langle k\rangle}{\langle n, n, k\rangle} \frac{\langle l\rangle}{\langle l, k, n\rangle} \delta(k ; n, n)^{5} \delta(l ; k, n)^{6}
$$

Now, let us close up to a tetrahedron. Taking into account two half-twists, we obtain that

$$
\left\langle K_{0}, n\right\rangle=\mu(n)^{-18} \sum_{k, l} \frac{\langle k\rangle}{\langle n, n, k\rangle} \frac{\langle l\rangle}{\langle l, k, n\rangle} \delta(k ; n, n)^{4} \delta(l ; k, n)^{7}\left\langle\begin{array}{ccc}
n & n & k \\
n & l & k
\end{array}\right\rangle
$$


Now, let us define the renormalized trihedron and tetrahedron coefficients $\langle\cdot\rangle^{\prime}$ by:

$$
\begin{aligned}
\left\langle\begin{array}{lll}
A & B & E \\
D & C & F
\end{array}\right\rangle & =\frac{1}{[A] ![B] ![C] ![D] ![E] ![F] !}\left\langle\begin{array}{ccc}
A & B & E \\
D & C & F
\end{array}\right\rangle^{\prime} \\
\langle a, b, c\rangle & =\frac{1}{[a] ![b] ![c] !}\langle a, b, c\rangle^{\prime}
\end{aligned}
$$

Since the colors on the edges of the tetrahedron cancel the colors of the edges of the trihedron, we obtain

$$
\left\langle K_{0}, n\right\rangle=\mu(n)^{-18} \sum_{k, l} \frac{\langle k\rangle}{\langle n, n, k\rangle^{\prime}} \frac{\langle l\rangle}{\langle l, k, n\rangle^{\prime}} \delta(k ; n, n)^{4} \delta(l ; k, n)^{7}\left\langle\begin{array}{ccc}
n & n & k \\
n & l & k
\end{array}\right\rangle^{\prime}
$$

In the above expression, $\mu(n)^{-18} \delta(k ; n, n)^{4} \delta(l ; k, n)^{7}$ is a monomial of $A$.

The $a_{i}$ and $b_{j}$ 's for the tetrahedron in question are given by:

$$
\begin{aligned}
a_{1}=a_{3} & =(2 n+k) / 2 \\
a_{2}=a_{4} & =(n+k+l) / 2 \\
b_{1} & =(n+2 k+l) / 2 \\
b_{2} & =(3 n+l) / 2 \\
b_{3} & =n+k .
\end{aligned}
$$

Since $a_{i}-b_{j}$ are the internal colors at the vertices of the tetrahedron, it follows that 6 quantum factorials cancel. In other words, we have:

$$
\frac{\langle k\rangle}{\langle n, n, k\rangle^{\prime}} \frac{\langle l\rangle}{\langle l, k, n\rangle^{\prime}} \prod_{i=1}^{3} \prod_{j=1}^{4}\left[b_{i}-a_{j}\right] !=\prod_{i=1}^{3}\left[b_{i}-a_{1}\right] ! \prod_{i=1}^{3}\left[b_{i}-a_{3}\right] !
$$

Combining the remaining 6 quantum factorials, and using the fact that

$$
J_{K}(n+1)=\frac{(-1)^{n}}{[n+1]}\langle K, n\rangle,
$$

the result follows.

Remark 1.2 follows from the fact that the quantum binomial coefficients are Laurent polynomials.

\section{The classical topology and geometry of $K_{0}$}

In this largely independent section, we give several facts about the classical geometry and topology of $K_{0}$. 


\subsection{The topology of $K_{0}$}

Torus knots can be embedded in closed surfaces of genus 1. Likewise, twisted torus knots can be embedded in closed surfaces of genus $2 . K_{0}$ is a $(1,1)$ knot, that is a special kind of a tunnel number 1 knot; see for example [GMM] and [MSY]. Consequently, its fundamental group has rank 2, i.e., is a 2-generator and 1-relator group, where the meridian is one of the generators. Note that 2bridge knots are also $(1,1)$ knots; thus from the point of view of presentation of the fundamental group, the knot $K$ looks similar to a 2-bridge knot, although it is not one. Dean studies which twisted torus knots are $(1,1)$ knots, De.

SnapPea reveals that the fundamental group of $K_{0}$ has the following presentation:

$$
\pi_{1}\left(S^{3}-K_{0}\right)=\langle a, b \mid a B a b a B a b b a B a B a b b a B a B a b b\rangle
$$

where the canonical meridian-longitude pair $(m, l)$ is given by

$$
(m, l)=(a, A B B A b A B B),
$$

where $A=a^{-1}$ and $B=b^{-1}$.

The Alexander polynomial of $K_{0}$ is:

$$
t^{-8}-t^{-7}+t^{-5}-t^{-4}+t^{-2}-t^{-1}+1-t+t^{2}-t^{4}+t^{5}-t^{7}+t^{8}
$$

and the sum of the absolute value of its coefficients is 13. By [GMM], and a private conversation with J. Rasmussen, 13 is also the rank of the Heegaard Floer Homology of Oszvath-Szabo; OS].

\subsection{The geometry of $K_{0}$}

SnapPea numerically computes the volume of $K_{0}$ to be:

$$
\operatorname{vol}\left(K_{0}\right)=3.474247 \ldots
$$

Of importance to us is the $A$-polynomial of $K_{0}$, which parametrizes the complex 1-dimensional part of the $\mathrm{SL}_{2}(\mathbb{C})$ character variety, restricted to the boundary of the knot complement. The $A$-polynomial of a knot was introduced by CCGLS, and is important in

(a) the study of deformations of the complete hyperbolic structure, 
(b) the study of slopes of essential surfaces in the knot complement with nonempty boundary, and

(c) in the shape of linear recursion relations of the colored Jones function, $\mathrm{Ga}$.

Since $K_{0}$ has rank 2, with a bit of work one can compute the $A$-polynomial of $K_{0}$, as was explained in [CCGLS. The method of [CCGLS, Section 7] assigns 2 by 2 matrices to the generators of the fundamental group, and uses resultants to compute the polynomial relation satisfied by the eigenvalues of the meridian and the longitude.

Alternatively, SnapPea provides an ideal triangulation of the knot complement of $K_{0}$, and one can write down the corresponding gluing equations and use elimination (via Groebner basis) to compute the polynomial relation satisifed by the eigenvalues of the meridian and the longitude. Boyd has developed a few more methods for computing the $A$-polynomial once its expected degree is known. Either method gives that $A(l, m)=(l-1) B\left(l, m^{2}\right)$ where $B(l, m)$ is an irreducible polynomial of $(l, m)$ of degree $10 \times 136$. Explicitly, we have:

$$
\begin{aligned}
& B(l, m)= \\
& \quad l^{10}+l^{9} m^{13}+l^{9} m^{14}-l^{8} m^{25}+4 l^{8} m^{26}-8 l^{8} m^{27}+3 l^{8} m^{28}-l^{8} m^{29}-3 l^{7} m^{40} \\
& -4 l^{7} m^{41}-l^{7} m^{42}+2 l^{6} m^{55}-2 l^{5} m^{66}+11 l^{5} m^{67}-6 l^{5} m^{68}+11 l^{5} m^{69}-2 l^{5} m^{70} \\
& +2 l^{4} m^{81}-l^{3} m^{94}-4 l^{3} m^{95}-3 l^{3} m^{96}-l^{2} m^{107}+3 l^{2} m^{108}-8 l^{2} m^{109}+4 l^{2} m^{110} \\
& -l^{2} m^{111}+l m^{122}+l m^{123}+m^{136} .
\end{aligned}
$$

We are interested in the 10 roots $\lambda_{k}(t)$ (so-called eigenvalues) of the equation

$$
B\left(\lambda, e^{i t}\right)=0
$$

We have that:

$$
B(l, 1)=(l+1)^{6}(l-1)^{4} .
$$

Since $B$ is reciprocal with real coefficients, it follows that if $\lambda_{k}(t)$ is an eigenvalue, so is the complex conjugate $\bar{\lambda}_{k}(t)$ and the inverse $1 / \lambda_{k}(t)$. Thus, the set of the eigenvalues has 4 -fold symmetry. It turns out that 6 eigenvalues $\lambda_{k}(t)$ for $t=5, \ldots, 10$ have magnitude 1 for all $t \in[0,2 \pi]$. The remaining four eigenvalues are

$$
\lambda_{1}(t), \quad \lambda_{2}(t)=\lambda_{1}(2 \pi-t), \quad \lambda_{3}(t)=\bar{\lambda}_{1}(t), \quad \lambda_{4}(t)=\bar{\lambda}_{1}(2 \pi-t),
$$

where $\lambda_{1}$ is chosen so that the slope of its magnitude at 0 is bigger than the slope of the magnitude of $\lambda_{2}$ at 0 . 
The plot of the set $\left\{\log \left|\lambda_{k}(t)\right| \mid k=1, \ldots, 10\right\}$ versus $t$ is:

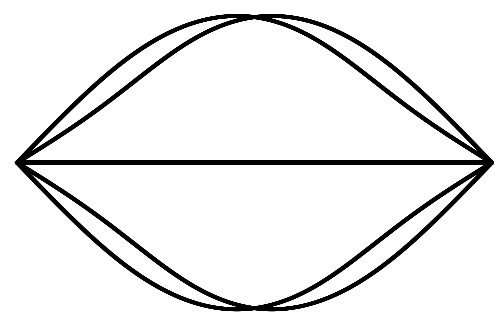

Collision of eigenvalues occurs only at $t=0, t=\pi$ and $t=2 \pi$, as follows from computing the roots of the discriminant $\operatorname{Disc}_{l} B(l, m)$ which lie on the unit circle.

Using the formula for the variation of the volume in terms of the $A$-polynomial, it follows that

$$
\int_{0}^{\pi} \log \left|\lambda_{1}(t)\right| d t=V_{1}+V_{3} \quad \int_{0}^{\pi} \log \left|\lambda_{2}(t)\right| d t=V_{2}-V_{3}
$$

where

$$
V_{1}=3.474247 \ldots, \quad V_{2}=1.962737 \ldots, \quad V_{3}=0.490684 \ldots
$$

Here, $V_{i}=\operatorname{vol}\left(\rho_{i}\right)$ for $i=1,2,3$ where

- $\rho_{1}$ is the discrete faithful representation, passing through $(m, l)=(1,-1)$.

- $\rho_{2}$ is another solution, passing though $(m, l)=(1,1)$,

- $\rho_{3}$ is a discrete faithful of the hyperbolic Dehn filling $K(2,0)$.

Using Snap, Boyd informs us that the invariant trace fields $E_{i}$ of $\rho_{i}$ are of type

- $6,[4,1],-753079$ for $\rho_{1}$, (that is, of degree 6 , with 4 real embeddings, 1 complex embedding and conductor -753079),

- $4,[2,1],-283$ for $\rho_{2}$ and $\rho_{3}$.

It is a coincidence that $E_{2}=E_{3}$, which implies that $V_{3}=V_{2} / 4$.

The entropy of a knot was defined in GG. Using the above information, it follows that the possible values of the entropy (at $t=2 \pi$ ) are given by the areas of the following curves:
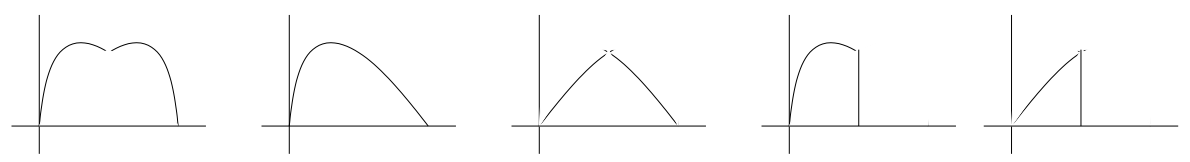

(or their reflection about $t=\pi$ ). These values are, respectively:

$$
2\left(V_{1}+V_{3}\right)=7.929864 \ldots
$$




$$
\begin{aligned}
V_{1}+V_{2} & =5.436985 \ldots \\
2\left(V_{2}-V_{3}\right) & =2.94411 \ldots \\
V_{1}+V_{3} & =3.964932 \ldots \\
V_{2}-V_{3} & =1.472053 \ldots
\end{aligned}
$$

Notice that none of these values equals to the volume $V_{1}$ of $K_{0}$.

\section{Towards the AJ conjecture for the knot $K_{0}$}

It was proven in GL that the sequence $J_{K}(n)$ for $n=0,1,2, \ldots$ of Laurent polynomials is $q$-holonomic, that is it satisfies a recursion relation.

Actually, it was explained in GL that the colored Jones function of any knot is given as a multisum of a $q$-proper hypergeometric function. Using the general theory of Wilf-Zeilberger, it follows that such multisums always satisfy recursion relations.

In general, the multisums in GL use as many summation variables as the number of the crossings of the knot, which makes things impractical to compute. In our case, we may use Theorem 1] which is a triple sum only.

What can one say about a recursion relation for $J_{K_{0}}$ ? It was conjectured in Ga (as part of the AJ Conjecture) that $J_{K_{0}}$ satisfies a degree 11 recursion relation of the form:

$$
\sum_{j=0}^{11} a_{j}\left(q^{n}, q\right) J_{K_{0}}(n+j)=0
$$

where

- $a_{j}(u, v) \in \mathbb{Q}(u, v)$ are rational functions with rational coefficients,

- the recursion relation is a $q$-deformation of the $A$-polynomial. That is,

$$
\sum_{j=0}^{11} a_{j}\left(M^{2}, 1\right) L^{j}=A(L, M) .
$$

- $\sum_{j=0}^{11} a_{j}(Q, q) E^{j}$ is reciprocal in the sense that

$$
a_{j}(Q, q)=\epsilon_{j} a_{11-j}\left(q^{-11} Q^{-1}, q\right)
$$

for all $j=0, \ldots, 11$ where $\epsilon_{j}= \pm 1$.

These properties cut down a guess for a recursion relation considerably.

Using Theorem 1] we have computed explicitly $J_{K_{0}}(n)$ for $n=1, \ldots, 19$. It is a challenging question to guess a formula for the so-called noncommutative $A$ polynomial of the knot $K_{0}$. 


\subsection{Acknowledgement}

Many people contributed to the ideas and the computations of this paper. We wish to thank D. Boyd, A. Champanerkar and N. Dunfield (for counseling on the $A$-polynomial), T. Morley (for Mathematica tutoring), M. Thistlethwaite (for the symmetry of $K$ ) and TTQ. Le (for discussions on a triple sum formula for the colored Jones function).

\section{A The numerical computation of $\mathrm{VC}(n)$}

\section{A.1 A recursive computation of $\mathrm{VC}(n)$}

In this appendix, we discuss the numerical evaluation of the colored Jones polynomial $J(n+1):=J_{K_{0}}(n+1)$ at $q=q_{0}$, where $q_{0}=\exp \left(i \frac{2 \pi}{n+1}\right)$. Let us begin by writing the formula in Theorem 1 in a numerically more efficient form

$$
\begin{aligned}
& J(n+1)= \\
& \frac{1}{[n+1]} \sum_{k=0,2}^{2 n} \sum_{l=|n-k|, 2}^{n+k} q^{-\frac{3}{8}\left(2 k+k^{2}\right)+\frac{7}{8}\left(2 l+l^{2}\right)-\frac{51}{8}\left(2 n+n^{2}\right)} \frac{[k+1][l+1]\left[\frac{k}{2}\right] !}{\left[\frac{2 n+k}{2}+1, \frac{k}{2}\right] !} Z,
\end{aligned}
$$

with $Z=$

$$
\begin{aligned}
& \sum_{z}(-1)^{\frac{k}{2}+z}\left[\begin{array}{c}
\frac{k+l-n}{2} \\
\frac{n+2 k+l}{2}-z
\end{array}\right]\left[\begin{array}{c}
\frac{n+l-k}{2} \\
\frac{3 n+l}{2}-z
\end{array}\right]\left[\begin{array}{c}
\frac{n+k-l}{2} \\
\frac{2 n+2 k}{2}-z
\end{array}\right]\left[\frac{2 n-k}{2}, z-\frac{n+k+l}{2}\right] ! \\
& {\left[z+1, \frac{n+k+l}{2}+1\right] ! }
\end{aligned}
$$

so that all the factors in $Z$ have $z$ dependence.

If we define $B=q^{1 / 2}$, then $Z$ in Equation (5) is an explicit rational function of $B$. We know that $J(n+1)$ is a finite sum with integer coefficients of integer powers of $q$, so it is also a finite sum of even powers of $B$. From Theorem 1 and Remark 1.2 we know that as a function of $B, J(n+1)(B)$ has an apparent second order pole at $B=B_{0}=\exp \left(i \frac{\pi}{n+1}\right)$. To verify the Volume Conjecture, we need to evaluate the Jones polynomial of the form:

$$
J(n+1)\left(B_{0}\right)=\left.\frac{f(B)}{\left(B-B_{0}\right)^{2}}\right|_{B=B_{0}},
$$

where $f(B)=g(B)\left(B-B_{0}\right)^{2}$ with $g(B)$ an analytic function at $B=B_{0}$. Apparently, $J(n+1)\left(B_{0}\right)=g\left(B_{0}\right)$. On the other hand

$$
g\left(B_{0}\right)=\left.\frac{1}{2} \frac{d^{2}}{d B^{2}} f(B)\right|_{B=B_{0}} .
$$


The next Equation will be the basis of our numerical computation:

$$
J(n+1)\left(B_{0}\right)=\left.\frac{1}{2} \frac{d^{2}}{d B^{2}} f(B)\right|_{B=B_{0}} .
$$

In other words, we have to separate the factor $\left(B-B_{0}\right)^{2}$ in the denominator in Equation (5), differentiate the rest part twice and evaluate at $B=B_{0}$. Considering the three summations and the complicated structure of the summand, we would expect that for large $n$, the computation load is huge. This is true if we employ symbolic manipulations using Mathematica or Maple, but numerically, we can do it much more efficiently if recursion relation is used both for the quantum numbers and their derivatives.

We have to evaluate the quantum integer, the quantum factorial and the quantum binomial coefficients etc. defined just before Theorem 1 Then the quantum integer is

$$
[n]=\frac{B^{n}-B^{-n}}{B-B^{-1}}
$$

which has first derivative

$$
[n]^{\prime}=\frac{n\left(B^{n-1}+B^{-n-1}\right)}{B-B^{-1}}-\frac{B^{n}-B^{-n}}{\left(B-B^{-1}\right)^{2}}\left(1+B^{-2}\right),
$$

and second derivative

$$
\begin{aligned}
{[n]^{\prime \prime}=} & \frac{n\left((n-1) B^{n-2}-(n+1) B^{-n-2}\right)}{B-B^{-1}}-\frac{2 n\left(B^{n-1}+B^{-n-1}\right)}{\left(B-B^{-1}\right)^{2}}\left(1+B^{-2}\right) \\
& +\frac{2\left(B^{n}-B^{-n}\right)}{\left(B-B^{-1}\right)^{3}}\left(1+3 B^{-2}\right) .
\end{aligned}
$$

In numerical calculations, three vectors may be used to store these values.

The quantum factorial satisfies the recursion relation

$$
[n] !=[n][n-1] !,
$$

which induces recursion relations for its first derivative

$$
[n] !^{\prime}=[n]^{\prime}[n-1] !+[n][n-1] !^{\prime}
$$

and second derivative

$$
[n] !^{\prime \prime}=[n]^{\prime \prime}[n-1] !+2[n]^{\prime}[n-1] !^{\prime}+[n][n-1] !^{\prime \prime} .
$$

Starting from $[1] !=1$, these factorials can be calculated simultaneously with the quantum integers and also stored in three vectors. 
Similarly, for quantum binomial coefficients, the recursion relations are

$$
\begin{gathered}
{\left[\begin{array}{l}
n \\
k
\end{array}\right]=B^{-k}\left[\begin{array}{c}
n-1 \\
k
\end{array}\right]+B^{n-k}\left[\begin{array}{l}
n-1 \\
k-1
\end{array}\right],} \\
{\left[\begin{array}{l}
n \\
k
\end{array}\right]=B^{-k}\left[\begin{array}{c}
n-1 \\
k
\end{array}\right]^{\prime}+B^{n-k}\left[\begin{array}{c}
n-1 \\
k-1
\end{array}\right]^{\prime}-k B^{-k-1}\left[\begin{array}{c}
n-1 \\
k
\end{array}\right]+(n-k) B^{n-k-1}\left[\begin{array}{l}
n-1 \\
k-1
\end{array}\right],}
\end{gathered}
$$

and $\left[\begin{array}{l}n \\ k\end{array}\right]=$

$$
\begin{aligned}
& B^{-k}\left[\begin{array}{c}
n-1 \\
k
\end{array}\right]^{\prime \prime}+B^{n-k}\left[\begin{array}{l}
n-1 \\
k-1
\end{array}\right]^{\prime \prime}-k B^{-k-1}\left[\begin{array}{c}
n-1 \\
k
\end{array}\right]^{\prime}+(n-k) B^{n-k-1}\left[\begin{array}{l}
n-1 \\
k-1
\end{array}\right]^{\prime} \\
& +k(k+1) B^{-k-2}\left[\begin{array}{c}
n-1 \\
k
\end{array}\right]+(n-k)(n-k-1) B^{n-k-2}\left[\begin{array}{c}
n-1 \\
k-1
\end{array}\right],
\end{aligned}
$$

with the starting condition $\left[\begin{array}{l}n \\ n\end{array}\right]=1$.

The recursion relations related to $[n, k]$ ! are given by

$$
\begin{gathered}
{[n, k] !=[n][n-1, k] !,} \\
{[n, k] !^{\prime}=[n]^{\prime}[n-1, k] !+[n][n-1, k] !^{\prime},}
\end{gathered}
$$

and

$$
[n, k] !^{\prime}=[n]^{\prime \prime}[n-1, k] !+[n][n-1, k] !^{\prime \prime}+[n]^{\prime}[n-1, k] !^{\prime},
$$

with starting condition $[k, k] !=1$. Notice that for the quantum binomial coefficients $\left[\begin{array}{l}n \\ k\end{array}\right]$ and $[n, k] !, n \geq k$ has to be satisfied. So, their numerical values can be put together to form a square matrix.

We need to take special care of the denominator $D$ in each summand. It has the form

$$
D=\left[\frac{2 n+k}{2}+1, \frac{k}{2}\right] ![n+1]=\left[\frac{2 n+k}{2}+1, n+1\right] !\left[n, \frac{k}{2}\right] ! P,
$$

where $P=[n+1]^{2}$. Note that $D$ is factorized to three factors. The first two with their first and second derivatives are readily evaluated by previous calculations and do not have $q=q_{0}$ as zeros. All the zero factors are contained in the third factor P. We have to extract the factor $B-B_{0}$ and evaluate the rest. we may write

$$
[n+1]=\frac{B^{n+1}-B^{-n-1}}{B-B^{-1}}=T S\left(B-B_{0}\right),
$$


where

$$
T=\frac{B^{-n-1}}{B-B^{-1}}, \quad S=B^{2 n+1}+B^{2 n} B_{0}+\cdots+B_{0}^{2 n+1} .
$$

It is straight forward to compute that

$$
\begin{aligned}
S\left(B_{0}\right) & =2(n+1) B_{0}^{-1} \\
S^{\prime}\left(B_{0}\right) & =(n+1)(2 n+1) B_{0}^{-2} \\
S^{\prime \prime}\left(B_{0}\right) & =\frac{4}{3} n(n+1)(2 n+1) B_{0}^{-3}
\end{aligned}
$$

and

$$
\begin{aligned}
T^{\prime}(B)= & \frac{-(n+1) B^{-n-2}}{B-B^{-1}}-\frac{B^{-n-1}}{\left(B-B^{-1}\right)^{2}}\left(1+B^{-2}\right) \\
T^{\prime \prime}(B)= & \frac{(n+1)(n+2) B^{-n-3}}{B-B^{-1}}+\frac{2(n+1) B^{-n-2}}{\left(B-B^{-1}\right)^{2}}\left(1+B^{-2}\right) \\
& +\frac{2 B^{-n-1}}{\left(B-B^{-1}\right)^{3}}\left(1+3 B^{-2}\right) .
\end{aligned}
$$

Now, we are in a position to compute the first and second derivatives of the summand of Equation (5), by invoking the values which we have. Finally, $g\left(B_{0}\right)$ is computed by multiplication and addition of the terms.

\section{A.2 The multiprecision algorithm}

Using the above scheme, we can go up to $n \sim 200$ with double precision in Fortran. Beyond that, the calculation overflows. The sequence $\operatorname{VC}(n)$ decreases until $n \sim 60$, where we reach a minimum, then it starts to increase. Of course, this is only an illusion due to the numerical round-off error. We checked the magnitude of the terms in the sum, it could go beyond that of the final result, which suggests that there could be significant cancellations among terms and the precision of our calculation is below the required one. We have to use multi-precision algorithm.

We take advantage of the multiprecision program by Bailey $\mathrm{Ba}$ (available at http://crd.lbl.gov/ dhbailey/mpdist/). We used MPFUN90 (Fortran-90 arbitrary precision package) to do our calculation. Using 40 digits, the series reaches a minimum at $n=131$. With 80 digits, the summation can be easily done if $n \sim 250$. The results shown in section 2 are obtained with 80 digits. we also tried 200 digits and calculated values up to $n=550$. Beyond that, the computer ran out of memory. We believe that with larger memory, it is easy for us to go beyond $n=1000$. With the increase of digits, we can go higher 
and higher values of $n$ but with the price that the computation is done slower and slower.

All the calculations that we have done suggest that the results in section 2 is true with high credibility. We may use more digits to calculate the $\mathrm{VC}(n)$ to ever-higher precision if necessity arises.

\section{B The colored Jones function of $K_{0}$}

Using Theorem 1 we can compute the colored Jones function $J(n):=J_{K_{0}}(n)$ for $n=1, \ldots, 19$. The polynomials become large soon, and we list here the first few of them.

$$
\begin{aligned}
& J(1)=1 \\
& J(2)=-\frac{1}{q^{19}}+\frac{1}{q^{18}}-\frac{1}{q^{17}}+\frac{1}{q^{10}}+\frac{1}{q^{8}} \\
& J(3)= \\
& \frac{1}{q^{53}}\left(1-q+q^{2}-2 q^{4}+2 q^{5}-2 q^{7}+q^{8}+q^{9}-q^{10}+q^{12}-q^{13}-q^{14}+q^{15}-q^{17}\right. \\
& \left.+q^{19}-q^{20}-q^{21}+q^{22}-q^{24}+q^{25}-q^{27}+q^{28}-q^{30}+q^{31}+q^{34}+q^{37}\right) \\
& J(4)= \\
& \frac{1}{q^{100}}\left(-1+2 q^{2}-4 q^{4}+q^{5}+4 q^{6}-5 q^{8}+6 q^{10}-5 q^{12}+4 q^{14}+q^{15}-4 q^{16}+3 q^{18}\right. \\
& +q^{19}-3 q^{20}-q^{21}+2 q^{22}+q^{23}-q^{24}-2 q^{25}+q^{26}+q^{27}-q^{29}-q^{30}+q^{31}+q^{32} \\
& -q^{34}+q^{36}-q^{38}+q^{40}-q^{41}-q^{42}+2 q^{44}-q^{45}-q^{46}-q^{47}+2 q^{48}-q^{50}-q^{51} \\
& \left.+q^{52}-q^{54}+q^{56}-q^{58}+q^{60}-q^{62}+q^{64}-q^{66}+q^{68}+q^{72}+q^{76}\right) \\
& J(5)= \\
& \frac{1}{q^{160}}\left(2-q-q^{2}-2 q^{3}+5 q^{5}+q^{6}-3 q^{7}-6 q^{8}+7 q^{10}+4 q^{11}-3 q^{12}-8 q^{13}-2 q^{14}\right. \\
& +7 q^{15}+6 q^{16}-2 q^{17}-8 q^{18}-2 q^{19}+6 q^{20}+4 q^{21}-2 q^{22}-6 q^{23}-2 q^{24}+6 q^{25} \\
& +3 q^{26}-q^{27}-5 q^{28}-2 q^{29}+5 q^{30}+2 q^{31}-3 q^{33}-2 q^{34}+3 q^{35}+q^{36}+q^{37}-q^{38} \\
& -2 q^{39}+q^{40}+q^{42}+q^{43}-q^{44}-q^{46}+q^{48}+q^{49}-q^{51}-q^{52}+q^{54}+q^{56}-q^{57} \\
& -q^{58}-q^{60}+2 q^{61}-3 q^{65}+2 q^{66}+q^{68}+q^{69}-3 q^{70}+2 q^{71}-q^{72}+q^{73}+q^{74} \\
& -3 q^{75}+2 q^{76}-q^{77}+q^{78}+q^{79}-3 q^{80}+q^{81}-q^{82}+q^{83}+q^{84}-2 q^{85}+q^{86}
\end{aligned}
$$




$$
\begin{aligned}
& -q^{87}+q^{88}-2 q^{90}+q^{91}+q^{93}-2 q^{95}+q^{98}-q^{100}+q^{103}-q^{105}+q^{108}-q^{110} \\
& \left.+q^{113}-q^{115}+q^{118}+q^{123}+q^{128}\right) \\
& J(6)= \\
& \frac{1}{q^{235}}\left(-1+q^{3}+q^{4}-q^{7}-2 q^{8}-q^{9}+5 q^{11}+4 q^{12}-q^{13}-5 q^{14}-6 q^{15}-q^{16}\right. \\
& +6 q^{17}+9 q^{18}+2 q^{19}-6 q^{20}-9 q^{21}-4 q^{22}+4 q^{23}+9 q^{24}+6 q^{25}-5 q^{26}-8 q^{27} \\
& -4 q^{28}+3 q^{29}+8 q^{30}+3 q^{31}-4 q^{32}-7 q^{33}-3 q^{34}+4 q^{35}+7 q^{36}+2 q^{37}-3 q^{38} \\
& -6 q^{39}-3 q^{40}+3 q^{41}+5 q^{42}+2 q^{43}-2 q^{44}-3 q^{45}-3 q^{46}+q^{47}+3 q^{48}+2 q^{49} \\
& -q^{51}-2 q^{52}-3 q^{53}+q^{54}+2 q^{55}+2 q^{56}+q^{57}-q^{58}-4 q^{59}-2 q^{60}+q^{61}+3 q^{62} \\
& +3 q^{63}+q^{64}-3 q^{65}-3 q^{66}-2 q^{67}+2 q^{68}+4 q^{69}+2 q^{70}-3 q^{72}-3 q^{73}+2 q^{75} \\
& +2 q^{76}+2 q^{77}-q^{78}-2 q^{79}-q^{81}+q^{82}+q^{83}+q^{86}-q^{87}-q^{90}+q^{92}+q^{94} \\
& -q^{96}-q^{99}+q^{100}-q^{102}+2 q^{103}-q^{105}-q^{107}-2 q^{108}+2 q^{109}+q^{110}+q^{112} \\
& -q^{113}-2 q^{114}+q^{116}+q^{118}-q^{120}+q^{122}-q^{126}+q^{128}-q^{132}+q^{134}-q^{136} \\
& -q^{138}+q^{140}+q^{141}-q^{142}-q^{144}+q^{147}-q^{148}+q^{153}-q^{154}-q^{155}+q^{159} \\
& \left.-q^{161}+q^{165}-q^{167}+q^{171}-q^{173}+q^{177}-q^{179}+q^{183}+q^{189}+q^{195}\right) \\
& J(7)= \\
& \frac{1}{q^{323}}\left(1+q^{2}-3 q^{3}+3 q^{7}-q^{8}-5 q^{10}+3 q^{11}+2 q^{12}+q^{13}+3 q^{14}-6 q^{15}-5 q^{16}\right. \\
& -5 q^{17}+9 q^{18}+8 q^{19}+3 q^{20}+3 q^{21}-11 q^{22}-13 q^{23}-8 q^{24}+12 q^{25}+12 q^{26} \\
& +9 q^{27}+7 q^{28}-12 q^{29}-17 q^{30}-13 q^{31}+9 q^{32}+11 q^{33}+10 q^{34}+12 q^{35}-9 q^{36} \\
& -16 q^{37}-13 q^{38}+8 q^{39}+10 q^{40}+8 q^{41}+9 q^{42}-9 q^{43}-14 q^{44}-11 q^{45}+9 q^{46} \\
& +10 q^{47}+8 q^{48}+7 q^{49}-9 q^{50}-12 q^{51}-11 q^{52}+7 q^{53}+8 q^{54}+7 q^{55}+6 q^{56} \\
& -6 q^{57}-8 q^{58}-11 q^{59}+4 q^{60}+6 q^{61}+6 q^{62}+7 q^{63}-3 q^{64}-5 q^{65}-11 q^{66}-q^{67} \\
& +3 q^{68}+6 q^{69}+8 q^{70}+q^{71}-2 q^{72}-11 q^{73}-4 q^{74}-q^{75}+5 q^{76}+8 q^{77}+4 q^{78} \\
& +2 q^{79}-9 q^{80}-6 q^{81}-4 q^{82}+2 q^{83}+6 q^{84}+5 q^{85}+5 q^{86}-4 q^{87}-5 q^{88}-6 q^{89} \\
& -q^{90}+2 q^{91}+4 q^{92}+6 q^{93}-2 q^{95}-4 q^{96}-2 q^{97}-2 q^{98}+q^{99}+4 q^{100}+q^{101} \\
& -q^{103}-2 q^{105}-q^{109}+2 q^{111}+2 q^{113}-3 q^{116}-2 q^{117}+q^{118}+3 q^{120}+q^{121} \\
& +3 q^{122}-2 q^{123}-3 q^{124}-2 q^{126}+q^{127}+4 q^{129}-q^{131}+q^{132}-q^{133}-3 q^{135} \\
& +3 q^{136}-q^{137}-q^{138}+2 q^{139}+q^{140}+q^{141}-3 q^{142}+3 q^{143}-2 q^{144}-2 q^{145} \\
& +q^{146}+q^{147}+q^{148}-3 q^{149}+4 q^{150}-2 q^{151}-q^{152}+q^{153}+q^{154}+q^{155}-3 q^{156} \\
& +3 q^{157}-3 q^{158}-q^{159}+q^{160}+q^{161}+q^{162}-q^{163}+3 q^{164}-3 q^{165}-q^{166}-q^{170}
\end{aligned}
$$




$$
\begin{aligned}
& +3 q^{171}-2 q^{172}+q^{174}-q^{177}+q^{178}-2 q^{179}+q^{181}+q^{183}+q^{185}-2 q^{186} \\
& +q^{188}-q^{189}+q^{190}+q^{192}-2 q^{193}+q^{195}-q^{196}+q^{197}+q^{199}-2 q^{200}+q^{202} \\
& -q^{203}+q^{206}-2 q^{207}+q^{209}+q^{213}-2 q^{214}+q^{220}-q^{221}-q^{228}+q^{233}-q^{235} \\
& \left.+q^{240}-q^{242}+q^{247}-q^{249}+q^{254}-q^{256}+q^{261}+q^{268}+q^{275}\right)
\end{aligned}
$$

\section{References}

[Ba] D.H. Bailey, A Fortran-90 Based Multiprecision System, ACM Transactions on Mathematical Software, 21 (1995) 379-387.

[Bo] W. Boyd, Mahler's measure and invariants of hyperbolic manifolds, Number theory for the millennium, I (Urbana, IL, 2000), 127-143, A K Peters, Natick, 2002. MathReview

[CDW] P. Callahan, J. Dean and J. Weeks, The simplest hyperbolic knots, J. Knot Theory Ramifications 8 (1999) 279-297. MathReview

[CCGLS] D. Cooper, M. Culler, H. Gillet, D. Long and P. Shalen, Plane curves associated to character varieties of 3-manifolds, Invent. Math. 118 (1994) 47-84. MathReview

[CG] O. Costin and S. Garoufalidis, in preparation.

[De] J. Dean, Small Seifert-fibered Dehn surgery on hyperbolic knots, Algebr. Geom. Topol. 3 (2003) 435-472. MathReview

[GL] S. Garoufalidis and TTQ. Le, The colored Jones function is q-holonomic arXiv:math.GT/0309214

[Ga] S. Garoufalidis, On the characteristic and deformation varieties of a knot, in: Proceedings of the CassonFest (C. Gordon and Y, Rieck, editors) Geometry and Topology Monographs 7 (2004) 291-309.

[GG] S. Garoufalidis and J. Geronimo, Asymptotics of q-difference equations, arXiv:math.QA/0405331

[Ge] W. Gehrke, Fortran 90 Language Guide, Springer, New York, 1995.

[GMM] H. Goda, H. Matsuda and T. Morifuji, Knot Floer homology of $(1,1)$-knots, arXiv:math.GT/0311084

[Gu] S. Gukov, Three-Dimensional Quantum Gravity, Chern-Simons Theory, and the A-Polynomial, arXiv:hep-th/0306165

[K] R. Kashaev, The hyperbolic volume of knots from the quantum dilogarithm, Modern Phys. Lett. A 39 (1997) 269-275. MathReview

[KL] L. Kauffman and S. Lins, Temperley-Lieb recoupling theory and invariants of 3-manifolds, Annals of Mathematics Studies 134, Princeton University Press, 1994. MathReview 
[J] V. Jones, Hecke algebra representation of braid groups and link polynomials, Annals Math. 126 (1987) p. 335-388. MathReview

[Le] T. Le, The Colored Jones Polynomial and the A-Polynomial of Two-Bridge Knots, arXiv:math.GT/0407521

[MV] G. Masbaum and P. Vogel, 3-valent graphs and the Kauffman bracket, Pacific J. Math. 164 (1994) 361-381. MathReview

[MSY] K. Morimoto, M. Sakuma and Y. Yokota, Identifying tunnel number one knots, J. Math. Soc. Japan 48 (1996) 667-688. MathReview

[MM] H. Murakami and J. Murakami, The colored Jones polynomials and the simplicial volume of a knot, Acta Math. 186 (2001) 85-104. MathReview

[M] H. Murakami, The asymptotic behavior of the colored Jones function of a knot and its volume, arXiv:math.GT/0004036

[OS] P. Oszvath and S. Szabo, Heegaard Floer homology and alternating knots, Geom. Topol. 7 (2003) 225-254. MathReview

[Sn] SnapPea and Snap software, http://www.geometrygames.org/SnapPea and http://www.ms.unimelb.edu.au/ snap

[Th] W. Thurston, The geometry and topology of 3-manifolds, 1979 notes, available from MSRI, http://www.msri.org/publications/books/gt3m/

[Tu] V. Turaev, The Yang-Baxter equation and invariants of links, Inventiones Math. 92 (1988) 527-553. MathReview

[WZ] H. Wilf and D. Zeilberger, An algorithmic proof theory for hypergeometric (ordinary and q) multisum/integral identities, Inventiones Math. 108 (1992) 575633. MathReview

School of Mathematics, Georgia Institute of Technology

Atlanta, GA 30332-0160, USA

and

School of Physics, Georgia Institute of Technology

Atlanta, GA 30332-0160, USA

Email: stavros@math.gatech.edu, gte158y@mail.gatech.edu

URL: http://www.math.gatech.edu/ stavros,

http://cns.physics.gatech.edu/ y-lan

Received: 16 December 2004 Revised: 21 April 2005 